\title{
Lengths of words in transformation semigroups generated by digraphs
}

\author{
P. J. Cameron ${ }^{1}$ - A. Castillo-Ramirez ${ }^{2}$ - \\ M. Gadouleau ${ }^{2}$ - J. D. Mitchell ${ }^{1}$
}

Received: 2 February 2016 / Accepted: 25 July 2016 / Published online: 8 August 2016 (C) The Author(s) 2016. This article is published with open access at Springerlink.com

\begin{abstract}
Given a simple digraph $D$ on $n$ vertices (with $n \geq 2$ ), there is a natural construction of a semigroup of transformations $\langle D\rangle$. For any edge $(a, b)$ of $D$, let $a \rightarrow b$ be the idempotent of rank $n-1$ mapping $a$ to $b$ and fixing all vertices other than $a$; then, define $\langle D\rangle$ to be the semigroup generated by $a \rightarrow b$ for all $(a, b) \in E(D)$. For $\alpha \in\langle D\rangle$, let $\ell(D, \alpha)$ be the minimal length of a word in $E(D)$ expressing $\alpha$. It is well known that the semigroup $\operatorname{Sing}_{n}$ of all transformations of rank at most $n-1$ is generated by its idempotents of rank $n-1$. When $D=K_{n}$ is the complete undirected graph, Howie and Iwahori, independently, obtained a formula to calculate $\ell\left(K_{n}, \alpha\right)$, for any $\alpha \in\left\langle K_{n}\right\rangle=\operatorname{Sing}_{n}$; however, no analogous non-trivial results are known when $D \neq K_{n}$. In this paper, we characterise all simple digraphs $D$ such that either $\ell(D, \alpha)$ is equal to Howie-Iwahori's formula for all $\alpha \in\langle D\rangle$, or $\ell(D, \alpha)=n-\operatorname{fix}(\alpha)$ for all $\alpha \in\langle D\rangle$, or $\ell(D, \alpha)=n-\operatorname{rk}(\alpha)$ for all $\alpha \in\langle D\rangle$. We also obtain bounds for $\ell(D, \alpha)$ when $D$ is an acyclic digraph or a strong tournament (the latter case corresponds to a smallest generating set of idempotents of rank $n-1$ of $\operatorname{Sing}_{n}$ ). We finish the paper with a list of conjectures and open problems.
\end{abstract}

Keywords Transformation semigroup · Simple digraph · Word length

\section{Introduction}

For any $n \in \mathbb{N}, n \geq 2$, let $\operatorname{Sing}_{n}$ be the semigroup of all singular (i.e. non-invertible) transformations on $[n]:=\{1, \ldots, n\}$. It is well known (see [2]) that $\operatorname{Sing}_{n}$ is generated

\footnotetext{
$凶 \quad$ A. Castillo-Ramirez acr8080@gmail.com

1 School of Mathematics and Statistics, University of St Andrews, St Andrews, Fife KY16 9SS, UK

2 School of Engineering and Computing Sciences, Durham University, South Road, Durham DH1 3LE, UK
} 
Fig. $1 \vec{T}_{5}$

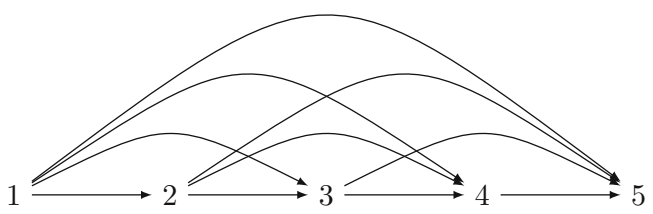

by its idempotents of defect 1 (i.e. the transformations $\alpha \in \operatorname{Sing}_{n}$ such that $\alpha^{2}=\alpha$ and $\operatorname{rk}(\alpha):=|\operatorname{Im}(\alpha)|=n-1)$. There are exactly $n(n-1)$ such idempotents, and each one of them may be written as $(a \rightarrow b)$, for $a, b \in[n], a \neq b$, where, for any $v \in[n]$,

$$
(v)(a \rightarrow b):= \begin{cases}b & \text { if } v=a, \\ v & \text { otherwise }\end{cases}
$$

Motivated by this notation, we refer to these idempotents as arcs.

In this paper, we explore the natural connections between simple digraphs on $[n]$ and subsemigroups of $\operatorname{Sing}_{n}$. For any subset $U \subseteq \operatorname{Sing}_{n}$, denote by $\langle U\rangle$ the semigroup generated by $U$. For any simple digraph $D$ with vertex set $V(D)=[n]$ and edge set $E(D)$, we associate the semigroup

$$
\langle D\rangle:=\left\langle(a \rightarrow b) \in \operatorname{Sing}_{n}:(a, b) \in E(D)\right\rangle
$$

We say that a subsemigroup $S$ of $\operatorname{Sing}_{n}$ is arc-generated by a simple digraph $D$ if $S=\langle D\rangle$.

For the rest of the paper, we use the term 'digraph' to mean 'simple digraph' (i.e. a digraph with no loops or multiple edges). A digraph $D$ is undirected if its edge set is a symmetric relation on $V(D)$, and it is transitive if its edge set is a transitive relation on $V(D)$. We shall always assume that $D$ is connected (i.e. for every pair $u, v \in V(D)$ there is either a path from $u$ to $v$, or a path from $v$ to $u$ ) because otherwise $\langle D\rangle \cong\left\langle D_{1}\right\rangle \times \cdots \times\left\langle D_{k}\right\rangle$, where $D_{1}, \ldots, D_{k}$ are the connected components of $D$. We say that $D$ is strong (or strongly connected) if for every pair $u, v \in V(D)$, there is a directed path from $u$ to $v$. We say that $D$ is a tournament if for every pair $u, v \in V(D)$ we have $(u, v) \in E(D)$ or $(v, u) \in E(D)$, but not both.

Many famous examples of semigroups are arc-generated. Clearly, by the discussion of the first paragraph, Sing ${ }_{n}$ is arc-generated by the complete undirected graph $K_{n}$. In fact, for $n \geq 3, \operatorname{Sing}_{n}$ is arc-generated by $D$ if and only if $D$ contains a strong tournament (see [3]). The semigroup of order-preserving transformations $\mathrm{O}_{n}:=\{\alpha \in$ $\left.\operatorname{Sing}_{n}: u \leq v \Rightarrow u \alpha \leq v \alpha\right\}$ is arc-generated by an undirected path $P_{n}$ on $[n]$, while the Catalan semigroup $\mathrm{C}_{n}:=\left\{\alpha \in \operatorname{Sing}_{n}: v \leq v \alpha, u \leq v \Rightarrow u \alpha \leq v \alpha\right\}$ is arcgenerated by a directed path $\vec{P}_{n}$ on $[n]$ (see [9, Corollary 4.11]). The semigroup of non-decreasing transformations $\mathrm{OI}_{n}:=\left\{\alpha \in \operatorname{Sing}_{n}: v \leq v \alpha\right\}$ is arc-generated by the transitive tournament $\vec{T}_{n}$ on [n] (Fig. 1 illustrates $\vec{T}_{5}$ ).

Connections between subsemigroups of $\operatorname{Sing}_{n}$ and digraphs have been studied before (see [9-12]). The following definition, which we shall adopt in the following sections, appeared in [12]: 
Definition 1 For a digraph $D$, the closure $\bar{D}$ of $D$ is the digraph with vertex set $V(\bar{D}):=V(D)$ and edge set $E(\bar{D}):=E(D) \cup\{(a, b):(b, a) \in E(D)$ is in a directed cycle of $D\}$.

Say that $D$ is closed if $D=\bar{D}$. Observe that $\langle D\rangle=\langle\bar{D}\rangle$ for any digraph $D$.

Recall that the orbits of $\alpha \in \operatorname{Sing}_{n}$ are the connected components of the digraph on $[n]$ with edges $\{(x, x \alpha): x \in[n]\}$. In particular, an orbit $\Omega$ of $\alpha$ is called cyclic if it is a cycle with at least two vertices. An element $x \in[n]$ is a fixed point of $\alpha$ if $x \alpha=x$. Denote by $\operatorname{cycl}(\alpha)$ and $\operatorname{fix}(\alpha)$ the number of cyclic orbits and fixed points of $\alpha$, respectively. Denote by $\operatorname{ker}(\alpha)$ the partition of $[n]$ induced by the kernel of $\alpha$ (i.e. the equivalence relation $\left.\left\{(x, y) \in[n]^{2}: x \alpha=y \alpha\right\}\right)$.

We introduce some further notation. For any digraph $D$ and $v \in V(D)$, define the in-neighbourhood and the out-neighbourhood of $v$ by

$N^{-}(v):=\{u \in V(D):(u, v) \in E(D)\}$ and $N^{+}(v):=\{u \in V(D):(v, u) \in E(D)\}$,

respectively. We extend these definitions to any subset $C \subseteq V(D)$ by letting $N^{\epsilon}(C):=$ $\bigcup_{c \in C} N^{\epsilon}(c)$, where $\epsilon \in\{+,-\}$. The in-degree and out-degree of $v$ are $\operatorname{deg}^{-}(v):=$ $\left|N^{-}(v)\right|$ and $\operatorname{deg}^{+}(v):=\left|N^{+}(v)\right|$, respectively, while the degree of $v$ is $\operatorname{deg}(v):=$ $\left|N^{-}(v) \cup N^{+}(v)\right|$. For any two vertices $u, v \in V(D)$, the $D$-distance from $u$ to $v$, denoted by $d_{D}(u, v)$, is the length of a shortest path from $u$ to $v$ in $D$, provided that such a path exists. The diameter of $D$ is $\operatorname{diam}(D):=\max \left\{d_{D}(u, v): u, v \in\right.$ $V(D), d_{D}(u, v)$ is defined .

Let $D$ be any digraph on $[n]$. We are interested in the lengths of transformations of $\langle D\rangle$ viewed as words in the free monoid $D^{*}:=\{(a \rightarrow b):(a, b) \in E(D)\}^{*}$. Say that a word $\omega \in D^{*}$ expresses (or evaluates to) $\alpha \in\langle D\rangle$ if $\alpha=\omega \phi$, where $\phi: D^{*} \rightarrow\langle D\rangle$ is the evaluation semigroup morphism. For any $\alpha \in\langle D\rangle$, let $\ell(D, \alpha)$ be the minimum length of a word in $D^{*}$ expressing $\alpha$. For $r \in[n-1]$, denote

$$
\begin{aligned}
\ell(D, r) & :=\max \{\ell(D, \alpha): \alpha \in\langle D\rangle, \operatorname{rk}(\alpha)=r\}, \\
\ell(D) & :=\max \{\ell(D, \alpha): \alpha \in\langle D\rangle\} .
\end{aligned}
$$

The main result in the literature in the study of $\ell(D, \alpha)$ was obtained by Howie and Iwahori, independently, when $D=K_{n}$.

Theorem 1.1 [4,5] For any $\alpha \in \operatorname{Sing}_{n}$,

$$
\ell\left(K_{n}, \alpha\right)=n+\operatorname{cycl}(\alpha)-\operatorname{fix}(\alpha)
$$

Therefore, $\ell\left(K_{n}, r\right)=n+\left\lfloor\frac{1}{2}(r-2)\right\rfloor$, for any $r \in[n-1]$, and $\ell\left(K_{n}\right)=\ell\left(K_{n}, n-\right.$ $1)=\left\lfloor\frac{3}{2}(n-1)\right\rfloor$.

In the following sections, we study $\ell(D, \alpha), \ell(D, r)$, and $\ell(D)$, for various classes of digraphs. In Sect. 2, we characterise all digraphs $D$ on $[n]$ such that either $\ell(D, \alpha)=$ $n+\operatorname{cycl}(\alpha)-\operatorname{fix}(\alpha)$ for all $\alpha \in\langle D\rangle$, or $\ell(D, \alpha)=n-\operatorname{fix}(\alpha)$ for all $\alpha \in\langle D\rangle$, or $\ell(D, \alpha)=n-\operatorname{rk}(\alpha)$ for all $\alpha \in\langle D\rangle$. In Sect. 3, we are interested in the maximal possible length of a transformation in $\langle D\rangle$ of rank $r$ among all digraphs $D$ on $[n]$ of 
certain class $\mathcal{C}$; we denote this number by $\ell_{\text {max }}^{\mathcal{C}}(n, r)$. In particular, when $\mathcal{C}$ is the class of acyclic digraphs, we find an explicit formula for $\ell_{\max }^{\mathcal{C}}(n, r)$. When $\mathcal{C}$ is the class of strong tournaments, we find upper and lower bounds for $\ell_{\max }^{\mathcal{C}}(n, r)$ (and for the analogously defined $\left.\ell_{\min }^{\mathcal{C}}(n, r)\right)$. Finally, in Sect. 4 we provide a list of conjectures and open problems.

\section{Arc-generated semigroups with short words}

Let $D$ be a digraph on $[n], n \geq 3$, and $\alpha \in\langle D\rangle$. Theorem 1.1 implies the following three bounds:

$$
\ell(D, \alpha) \geq n+\operatorname{cycl}(\alpha)-\operatorname{fix}(\alpha) \geq n-\operatorname{fix}(\alpha) \geq n-\operatorname{rk}(\alpha) .
$$

The lowest bound is always achieved for constant transformations (i.e. transformations of rank 1).

Lemma 2.1 For any digraph $D$ on $[n]$, if $\alpha \in\langle D\rangle$ has rank 1 , then $\ell(D, \alpha)=n-1$.

Proof It is clear that $\ell(D, \alpha) \geq n-1$ because $\alpha$ has $n-1$ non-fixed points. Let $\operatorname{Im}(\alpha)=\left\{v_{0}\right\} \subseteq[n]$. Note that, for any $v \in[n]$, there is a directed path in $D$ from $v$ to $v_{0}$ (as otherwise, $\alpha \notin\langle D\rangle$ ). For any $d \geq 1$, let

$$
C_{d}:=\left\{v \in[n]: d_{D}\left(v, v_{0}\right)=d\right\} .
$$

Clearly, $[n] \backslash\left\{v_{0}\right\}=\bigcup_{d=1}^{m} C_{d}$, where $m:=\max _{v \in[n]}\left\{d_{D}\left(v, v_{0}\right)\right\}$ and the union is disjoint. For any $v \in C_{d}$, let $v^{\prime}$ be a vertex in $C_{d-1}$ such that $\left(v \rightarrow v^{\prime}\right) \in D$. For any distinct $v, u \in C_{d}$ and any choice of $v^{\prime}, u^{\prime} \in C_{d-1}$, the $\operatorname{arcs}\left(v \rightarrow v^{\prime}\right)$ and $\left(u \rightarrow u^{\prime}\right)$ commute; hence, we can decompose $\alpha$ as

$$
\alpha=\bigcirc_{d=m}^{1} \bigcirc_{v \in C_{d}}\left(v \rightarrow v^{\prime}\right),
$$

where the composition of arcs is done from $m$ down to 1 .

Remark 1 Using a similar argument as in the previous proof, we may show that $\langle D\rangle$ contains all constant transformations if and only if $D$ is strongly connected.

Inspired by the bounds given in (1), we characterise all the connected digraphs $D$ on $[n]$ satisfying the following conditions:

$$
\begin{gathered}
\forall \alpha \in\langle D\rangle, \ell(D, \alpha)=n+\operatorname{cycl}(\alpha)-\operatorname{fix}(\alpha) ; \\
\forall \alpha \in\langle D\rangle, \ell(D, \alpha)=n-\operatorname{fix}(\alpha) ; \\
\forall \alpha \in\langle D\rangle, \ell(D, \alpha)=n-\operatorname{rk}(\alpha) .
\end{gathered}
$$




\subsection{Digraphs satisfying condition (C1)}

Theorem 1.1 says that $K_{n}$ satisfies (C1). In order to characterise all digraphs satisfying (C1), we introduce the following property on a digraph $D$ :

( $\star$ If $d_{D}\left(v_{0}, v_{2}\right)=2$ and $v_{0}, v_{1}, v_{2}$ is a directed path in $D$, then $N^{+}\left(\left\{v_{1}, v_{2}\right\}\right) \subseteq$ $\left\{v_{0}, v_{1}, v_{2}\right\}$.

We shall study the strong components of digraphs satisfying property $(\star)$. We state few observations that we use repeatedly in this section.

Remark 2 Suppose that $D$ satisfies property ( $\star$ ). If $v_{0}, v_{1}, v_{2}$ is a directed path in $D$ and $\operatorname{deg}^{+}\left(v_{1}\right)>2$, or deg $^{+}\left(v_{2}\right)>2$, then $\left(v_{0}, v_{2}\right) \in E(D)$. Indeed, if $\left(v_{0}, v_{2}\right) \notin E(D)$, then $d_{D}\left(v_{0}, v_{2}\right)=2$, so, by property $(\star), N^{+}\left(\left\{v_{1}, v_{2}\right\}\right) \subseteq\left\{v_{0}, v_{1}, v_{2}\right\}$; this contradicts that $\operatorname{deg}^{+}\left(v_{1}\right)>2, \operatorname{ordeg}^{+}\left(v_{2}\right)>2$.

Remark 3 Suppose that $D$ satisfies property $(\star)$. If $v_{0}, v_{1}, v_{2}$ is a directed path in $D$ and either $v_{1}$ or $v_{2}$ has an out-neighbour not in $\left\{v_{0}, v_{1}, v_{2}\right\}$, then $\left(v_{0}, v_{1}\right) \in E(D)$.

Remark 4 If $D$ satisfies property $(\star)$, then $\operatorname{diam}(D) \leq 2$. Indeed, if $v_{0}, v_{1}, \ldots, v_{k}$ is a directed path in $D$ with $d_{D}\left(v_{0}, v_{k}\right)=k \geq 3$, then $v_{0}, v_{1}, v_{2}$ is a directed path in $D$ and $v_{2}$ has an out-neighbour $v_{3} \notin\left\{v_{0}, v_{1}, v_{2}\right\}$; by Remark $3,\left(v_{0}, v_{2}\right) \in E(D)$, which contradicts that $d_{D}\left(v_{0}, v_{k}\right)=k$.

Note that digraphs satisfying property $(\star)$ are a slight generalisation of transitive digraphs.

Let $D$ be a digraph and let $C_{1}$ and $C_{2}$ of be two strong components of $D$. We say that $C_{1}$ connects to $C_{2}$ if $\left(v_{1}, v_{2}\right) \in E(D)$ for some $v_{1} \in C_{1}, v_{2} \in C_{2}$; similarly, we say that $C_{1}$ fully connects to $C_{2}$ if $\left(v_{1}, v_{2}\right) \in E(D)$ for all $v_{1} \in C_{1}, v_{2} \in C_{2}$. The strong component $C_{1}$ is called terminal if there is no strong component $C \neq C_{1}$ of $D$ such that $C_{1}$ connects to $C$.

Lemma 2.2 Let $D$ be a closed digraph satisfying property $(\star)$. Then, any strong component of $D$ is either an undirected path $P_{3}$ or complete. Furthermore, $P_{3}$ may only appear as a terminal strong component of $D$.

Proof Let $C$ be a strong component of $D$. Since $D$ is closed, $C$ must be undirected. The lemma is clear if $|C| \leq 3$, so assume that $|C| \geq 4$. We have two cases:

Case 1 Every vertex in $C$ has degree at most 2. Then $C$ is a path or a cycle. Since $|C| \geq 4$ and $\operatorname{diam}(D) \leq 2$, then $C$ is a cycle of length 4 or 5 ; however, these cycles do not satisfy property $(\star)$.

Case 2 There exists a vertex $a \in C$ of degree 3 or more. Any two neighbours of $a$ are adjacent: indeed, for any $u, v \in N(a), u, a, v$ is a path and $\operatorname{deg}^{+}(a)>2$, so $(u, v) \in E(D)$ by Remark 2. Hence, the neighbourhood of $a$ is complete and every neighbour of $a$ has degree 3 or more. Applying this rule recursively, we obtain that every vertex in $C$ has degree 3 or more, and the neighbourhood of every vertex is complete. Therefore, $C$ is complete because $\operatorname{diam}(D) \leq 2$.

Finally, if $P_{3}$ is a strong component of $D$, there cannot be any edge coming out of it because of property $(\star)$, so it must be a terminal component. 
Lemma 2.3 Let $D$ be a closed digraph satisfying property ( $\star$ ). Let $C_{1}$ and $C_{2}$ be strong components of $D$, and suppose that $C_{1}$ connects to $C_{2}$.

(i) If $C_{2}$ is non-terminal, then $C_{1}$ fully connects to $C_{2}$.

(ii) Let $\left|C_{2}\right|=1$. If either $\left|C_{1}\right| \neq 2$, or the vertex in $C_{1}$ that connects to $C_{2}$ has out-degree at least 3 , then $C_{1}$ fully connects to $C_{2}$.

(iii) Let $\left|C_{2}\right|=2$. If not all vertices in $C_{1}$ connect to the same vertex in $C_{2}$, then $C_{1}$ fully connects to $C_{2}$.

(iv) If $\left|C_{2}\right| \geq 3$, then $C_{1}$ fully connects to $C_{2}$.

Proof Recall that $C_{1}$ and $C_{2}$ are undirected because $D$ is closed. If $\left|C_{1}\right|=1$ and $\left|C_{2}\right|=1$, clearly $C_{1}$ fully connects to $C_{2}$. Henceforth, we assume $\left|C_{1}\right| \geq 2$ or $\left|C_{2}\right| \geq 2$. Let $c_{1} \in C_{1}$ and $c_{2} \in C_{2}$ be such that $\left(c_{1}, c_{2}\right) \in E(D)$. As $C_{1}$ is a non-terminal, Lemma 2.2 implies that $C_{1}$ is complete.

(i) As $C_{2}$ is non-terminal, there exists $d \in D \backslash\left(C_{1} \cup C_{2}\right)$ such that $\left(c_{2}, d\right) \in E(D)$. Suppose that $\left|C_{1}\right| \geq 2$. Then, for any $c_{1}^{\prime} \in C_{1} \backslash\left\{c_{1}\right\}, c_{1}^{\prime}, c_{1}, c_{2}$ is a directed path in $D$ with $d \in N^{+}\left(c_{2}\right)$, so Remark 3 implies $\left(c_{1}^{\prime}, c_{2}\right) \in E(D)$. Suppose now that $\left|C_{2}\right| \geq 2$. Then, for any $c_{2}^{\prime} \in C_{2} \backslash\left\{c_{2}\right\}, c_{1}, c_{2}, c_{2}^{\prime}$ is a directed path in $D$ with $d \in N^{+}\left(c_{2}\right)$, so again $\left(c_{1}, c_{2}^{\prime}\right) \in E(D)$. Therefore, $C_{1}$ fully connects to $C_{2}$.

(ii) Suppose that $\left|C_{1}\right| \geq 2$. If $\left|C_{1}\right|>2$, then $\mathrm{deg}^{+}\left(c_{1}\right)>2$, because $C_{1}$ is complete. Thus, for each $c_{1}^{\prime} \in C_{1} \backslash\left\{c_{1}\right\}, c_{1}^{\prime}, c_{1}, c_{2}$ is a directed path in $D$ with $\operatorname{deg}^{+}\left(c_{1}\right)>2$, so $\left(c_{1}^{\prime}, c_{2}\right) \in E(D)$ by Remark 2 . As $\left|C_{2}\right|=1$, this shows that $C_{1}$ fully connects to $C_{2}$.

(iii) Let $C_{2}=\left\{c_{2}, c_{2}^{\prime}\right\}$ and let $c_{1}^{\prime} \in C_{1} \backslash\left\{c_{1}\right\}$ be such that $\left(c_{1}^{\prime}, c_{2}^{\prime}\right) \in E(D)$. For any $b, d \in C_{1}, b \neq c_{1}, d \neq c_{1}^{\prime}$, both $b, c_{1}, c_{2}$ and $d, c_{1}^{\prime}, c_{2}^{\prime}$ are directed paths in $D$ with $c_{2}^{\prime} \in N^{+}\left(c_{2}\right)$ and $c_{2} \in N^{+}\left(c_{2}^{\prime}\right)$; hence, $\left(b, c_{2}\right),\left(d, c_{2}^{\prime}\right) \in E(D)$ by Remark 3.

(iv) Suppose that $C_{2}=P_{3}$. Say $C_{2}=\left\{c_{2}, c_{2}^{\prime}, c_{2}^{\prime \prime}\right\}$ with either $d_{D}\left(c_{2}, c_{2}^{\prime \prime}\right)=2$ or $d_{D}\left(c_{2}^{\prime}, c_{2}^{\prime \prime}\right)=2$. In any case, $c_{1}, c_{2}, c_{2}^{\prime}$ is a directed path in $D$ with $c_{2}^{\prime \prime} \in$ $N^{+}\left(\left\{c_{2}, c_{2}^{\prime}\right\}\right)$, so $\left(c_{1}, c_{2}^{\prime}\right) \in E(D)$ by Remark 3; now, $c_{1}, c_{2}^{\prime}, c_{2}^{\prime \prime}$ is a directed path in $D$ with $c_{2} \in N^{+}\left(\left\{c_{2}^{\prime}, c_{2}^{\prime \prime}\right\}\right)$, so $\left(c_{1}, c_{2}^{\prime \prime}\right) \in E(D)$. Hence, $c_{1}$ is connected to all vertices of $C_{2}$. As $C_{1}$ is complete, a similar argument shows that every $c_{1}^{\prime} \in C_{1} \backslash\left\{c_{1}\right\}$ connects to every vertex in $C_{2}$.

Suppose now that $C_{2}=K_{m}$ for $m \geq 3$. By a similar reasoning as the previous paragraph, we show that $\left(c_{1}, v\right) \in E(D)$ for all $v \in C_{2}$. Now, for any $c_{1}^{\prime} \in C_{1} \backslash\left\{c_{1}\right\}, v \in C_{2}, c_{1}^{\prime}, c_{1}, v$ is a directed path in $D$ so $\left(c_{1}^{\prime}, v\right) \in E(D)$ by Remark 3.

Lemma 2.4 Let $D$ be a closed digraph satisfying property $(\star)$. Let $C_{i}, i=1,2,3$, be strong components of $D$, and suppose that $C_{1}$ connects to $C_{2}$ and $C_{2}$ connects to $C_{3}$. If $C_{1}$ does not connect to $C_{3}$, then $\left|C_{2}\right|=\left|C_{3}\right|=1, C_{3}$ is terminal in $D$, and $C_{2}$ is terminal in $D \backslash C_{3}$.

Proof By Lemma 2.3 (i), $C_{1}$ fully connects to $C_{2}$. Assume that $C_{1}$ does not connect to $C_{3}$. Let $c_{i} \in C_{i}, i=1,2,3$, be such that $\left(c_{1}, c_{2}\right),\left(c_{2}, c_{3}\right) \in E(D)$. If $C_{2}$ has a vertex different from $c_{2}$, Remark 3 ensures that $\left(c_{1}, c_{3}\right) \in E(D)$, which contradicts our hypothesis. Then $\left|C_{2}\right|=1$. The same argument applies if $C_{3}$ has a vertex different 
from $c_{3}$, so $\left|C_{3}\right|=1$. Finally, Remark 3 applied to the path $c_{1}, c_{2}, c_{3}$ also implies that $C_{3}$ is terminal in $D$ and $C_{2}$ is terminal in $D \backslash C_{3}$.

The following result characterises all digraphs satisfying condition (C1).

Theorem 2.5 Let $D$ be a connected digraph on $[n]$. The following are equivalent:

(i) For all $\alpha \in\langle D\rangle, \ell(D, \alpha)=n+\operatorname{cycl}(\alpha)-\operatorname{fix}(\alpha)$.

(ii) $D$ is closed satisfying property $(\star)$.

Proof In order to simplify notation, denote

$$
g(\alpha):=n+\operatorname{cycl}(\alpha)-\operatorname{fix}(\alpha) .
$$

First, we show that (i) implies (ii). Suppose $\ell(D, \alpha)=g(\alpha)$ for all $\alpha \in\langle D\rangle$. We use the one-line notation for transformations: $\alpha=(1) \alpha(2) \alpha \ldots(k) \alpha$, where $x=(x) \alpha$ for all $x>k, x \in[n]$. Clearly, if $D$ is not closed, there exists an arc $\alpha \in\langle D\rangle \backslash D$, so $1<\ell(D, \alpha) \neq g(\alpha)=1$. In order to prove that property $(\star)$ holds, let $1,2,3$ be a shortest path in $D$. If $(2 \rightarrow v) \in\langle D\rangle$, for some $v \in[n] \backslash\{1,2,3\}$, then $\alpha=3 v 3 v \in$ $\langle D\rangle$, but $g(\alpha)=2 \neq \ell(D, \alpha)=3$. If $(3 \rightarrow v) \in\langle D\rangle$, then $\alpha=3 v v v \in\langle D\rangle$, but $g(\alpha)=3 \neq \ell(D, \alpha)=4$. Therefore, $N^{+}(\{2,3\}) \subseteq\{1,2,3\}$, and ( $\left.\star\right)$ holds.

Conversely, we show that (ii) implies (i). Let $\alpha \in\langle D\rangle$. We remark that any cycle of $\alpha$ belongs to a strong component of $D$.

Claim 2.6 Let $C$ be a strong component of $D$. Then either $\alpha$ fixes all vertices of $C$ or $|(C \alpha) \cap C|<|C|$.

Proof Suppose that $\left.\alpha\right|_{C}$, the restriction of $\alpha$ to $C$, is non-trivial and $|(C \alpha) \cap C|=|C|$. Then $\left.\alpha\right|_{C}$ is a permutation of $C$. Let $u \in C$ and suppose that $(u \rightarrow v)$ is the first arc moving $u$ in a word expressing $\alpha$ in $D^{*}$. If $v \in C$, we have $u \alpha=v \alpha$, which contradicts that $\left.\alpha\right|_{C}$ is a permutation. If $v \in C^{\prime}$ for some other strong component $C^{\prime}$ of $D$, then $u \alpha \notin C$ which again contradicts our assumption.

Claim 2.7 Let $u, v \in[n]$ be such that $u \alpha=v$. If $d_{D}(u, v)=2$, then:

1. $v$ is in a terminal component of $D$.

2. There is a path $u, w, v$ of length 2 in $D$ such that $w \alpha=v \alpha=v$; for any other path $u, x, v$ of length 2 in $D$, we have $x \alpha \in\{x, v\}$.

Proof Let $C_{1}$ and $C_{2}$ be strong components of $D$ such that $u \in C_{1}$ and $v \in C_{2}$. We analyse the four possible cases in which $d_{D}(u, v)=2$. In the first three cases, we use the fact that $\left\langle P_{3}\right\rangle \cong \mathrm{O}_{3}$, hence we can order $u<w<v$ and $\alpha$ is an increasing transformation of the ordered set $\{u, w, v\}$; thus $u \alpha=w \alpha=v \alpha=v$.

Case $1 C_{1}=C_{2}$. By Lemma 2.2, $C_{1} \cong P_{3}$ and it is a terminal component. Therefore, 2. holds as there is a unique path from $u$ to $v$.

Case $2 C_{1}$ connects to $C_{2}$ and $\left|C_{2}\right| \neq 2$. As $d_{D}(u, v)=2, C_{1}$ does not fully connect $C_{2}$, so, by Lemma $2.3,\left|C_{2}\right|=1, C_{2}$ is terminal, $\left|C_{1}\right|=2$, and the vertex $w \in C_{1}$ connecting to $C_{2}=\{v\}$ has out-degree 2 . Then, by property $(\star)$, $u, w, v$ is the unique path from $u$ to $v$. 
Case $3 C_{1}$ connects to $C_{2}$ and $\left|C_{2}\right|=2$. As $d_{D}(u, v)=2, C_{1}$ does not fully connect $C_{2}$, so, by Lemma $2.3, C_{2}$ is terminal and $u, w, v$ is the unique path of length two from $u$ to $v$, where $w$ is the other vertex of $C_{2}$.

Case $4 C_{1}$ does not connect to $C_{2}$. Since $d_{D}(u, v)=2$, there exist strong components $C^{(1)}, \ldots, C^{(k)}$ such that $C_{1}$ connects to $C^{(i)}$ and $C^{(i)}$ connects to $C_{2}$, for all $1 \leq i \leq k$. By Lemma 2.4, $C^{(i)}=\left\{x_{i}\right\}, C_{2}=\{v\}$ is terminal and $N^{+}\left(x_{i}\right)=\{v\}$ for all $i$. Thus $u, x_{i}, v$ are the only paths of length two from $u$ to $v$; in particular, $x_{i} \alpha \in\left\{x_{i}, v\right\}$ for all $x_{i}$. As $u \alpha=v$, there must exist $1 \leq j \leq k$ such that $w:=x_{j}$ is mapped to $v$.

Now we produce a word $\omega \in D^{*}$ expressing $\alpha$ of length $g(\alpha)$. Define

$$
U:=\left\{u \in D: d_{D}(u, u \alpha)=2\right\} .
$$

For every $u \in U$, let $u^{\prime}$ be a vertex in $D$ such that $u, u^{\prime}, u \alpha$ is a path and $u^{\prime} \alpha=u \alpha$. The existence of $u^{\prime}$ is guaranteed by Claim 2.7. Define a word $\omega_{0} \in D^{*}$ by

$$
\omega_{0}:=\bigcirc u \in U\left(u \rightarrow u^{\prime}\right)\left(u^{\prime} \rightarrow u \alpha\right)
$$

Sort the strong components of $D$ in topological order: $C_{1}, \ldots, C_{k}$, i.e. for $i \neq j$, $C_{i}$ connects to $C_{j}$ only if $j>i$. For each $1 \leq i \leq k$, define

$$
S_{i}:=\left\{v \in C_{i} \backslash\left(U \cup U^{\prime}\right): v \alpha \in C_{i}\right\}
$$

where $U^{\prime}:=\left\{u^{\prime}: u \in U\right\}$, and consider the transformation $\beta_{i}: C_{i} \rightarrow C_{i}$ defined by

$$
x \beta_{i}= \begin{cases}x \alpha & \text { if } x \in S_{i} \\ x & \text { otherwise }\end{cases}
$$

If $\left|C_{i}\right| \leq 2$ or $C_{i} \cong P_{3}$, then $\operatorname{cycl}\left(\beta_{i}\right)=0$ and $\beta_{i}$ can be computed with $\left|C_{i}\right|-$ $\operatorname{fix}\left(\beta_{i}\right)$ arcs. Otherwise, $C_{i}$ is a complete undirected graph. If $\beta_{i} \in \operatorname{Sing}\left(C_{i}\right)$, then by Theorem 1.1, there is a word $\omega_{i} \in C_{i}^{*} \subseteq D^{*}$ of length $\left|C_{i}\right|+\operatorname{cycl}\left(\beta_{i}\right)-\operatorname{fix}\left(\beta_{i}\right)$ expressing $\beta_{i}$. Suppose now that $\beta_{i}$ is a non-identity permutation of $C_{i}$. By Claim 2.6, $\alpha$ does not permute $C_{i}$ and there exists $h_{i} \in C_{i} \backslash\left(C_{i} \alpha\right)$. Note that $h_{i} \in C_{i} \backslash S_{i}$. Define $\hat{\beta}_{i} \in \operatorname{Sing}\left(C_{i}\right)$ by

$$
x \hat{\beta}_{i}= \begin{cases}x \alpha & \text { if } x \in S_{i} \\ a_{i} & \text { if } x=h_{i} \\ x & \text { otherwise }\end{cases}
$$

where $a_{i}$ is any vertex in $S_{i}$. Then $\left.\alpha\right|_{S_{i}}=\left.\hat{\beta}\right|_{S_{i}}$. Again by Theorem 1.1 , there is a word $\omega_{i} \in C_{i}^{*} \subseteq D^{*}$ of length $\left|C_{i}\right|+\operatorname{cycl}\left(\hat{\beta}_{i}\right)-\operatorname{fix}(\hat{\beta})=\left|C_{i}\right|+\operatorname{cycl}\left(\beta_{i}\right)-\operatorname{fix}\left(\beta_{i}\right)$ expressing $\hat{\beta}_{i}$. 
The following word maps all the vertices in $[n] \backslash\left(U \cup U^{\prime} \cup C_{i}\right)$ that have image in $C_{i}$ :

$$
\omega_{i}^{\prime}=\bigcirc\left\{(a \rightarrow a \alpha): a \in[n] \backslash\left(U \cup U^{\prime} \cup C_{i}\right), a \alpha \in C_{i}\right\}
$$

Finally, let

$$
\omega:=\omega_{0} \omega_{k} \omega_{k}^{\prime} \ldots \omega_{1} \omega_{1}^{\prime} \in D^{*} .
$$

It is easy to check that $\omega$ indeed expresses $\alpha$. Since $\sum_{i=1}^{k} \operatorname{fix}\left(\beta_{i}\right)=\operatorname{fix}(\alpha)+$ $\sum_{i=1}^{k}\left|C_{i} \backslash S_{i}\right|$ and $\sum_{i=1}^{k} \ell\left(\omega_{i}^{\prime}\right)=\sum_{i=1}^{k}\left|C_{i} \backslash\left(U \cup U^{\prime} \cup S_{i}\right)\right|$, we have

$$
\ell(\omega)=2|U|+\sum_{i=1}^{k}\left(\ell\left(\omega_{i}\right)+\ell\left(\omega_{i}^{\prime}\right)\right)=n+\sum_{i=1}^{k} \operatorname{cycl}\left(\beta_{i}\right)-\operatorname{fix}(\alpha)=g(\alpha) .
$$

\subsection{Digraphs satisfying condition (C2)}

The characterisation of connected digraphs satisfying condition (C2) is based on the classification of connected digraphs $D$ such that $\operatorname{cycl}(\alpha)=0$, for all $\alpha \in\langle D\rangle$.

For $k \geq 3$, let $\Theta_{k}$ be the directed cycle of length $k$. Consider the digraphs $\Gamma_{1}, \Gamma_{2}, \Gamma_{3}$ and $\Gamma_{4}$ as illustrated below:

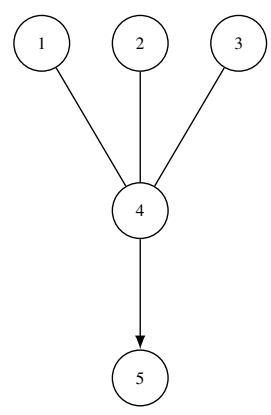

$\Gamma_{1}$

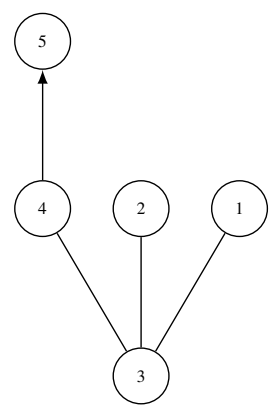

$\Gamma_{2}$

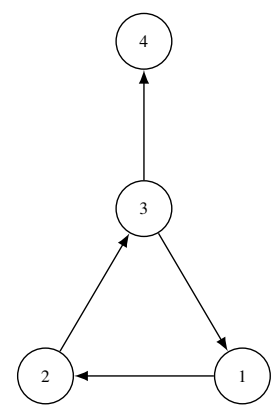

$\Gamma_{3}$

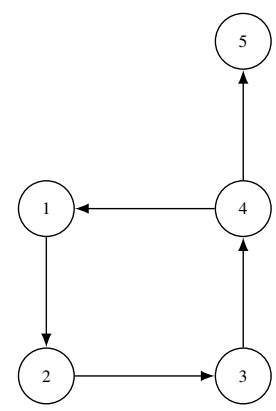

$\Gamma_{4}$

Lemma 2.8 Let $D$ be a connected digraph on $[n]$. The following are equivalent:

(i) For all $\alpha \in\langle D\rangle \operatorname{cycl}(\alpha)=0$.

(ii) $D$ has no subdigraph isomorphic to $\Gamma_{1}, \Gamma_{2}, \Gamma_{3}, \Gamma_{4}$, or $\Theta_{k}$, for all $k \geq 5$.

Proof In order to prove that (i) implies (ii), we show that if $\Gamma$ is equal to $\Gamma_{i}$ or $\Theta_{k}$, for $i \in[4], k \geq 5$, then there exists $\alpha \in\langle\Gamma\rangle \operatorname{such}$ that $\operatorname{cycl}(\alpha) \neq 0$. 
- If $\Gamma=\Gamma_{1}$, take

$$
\begin{aligned}
\alpha & :=(3 \rightarrow 4)(4 \rightarrow 5)(1 \rightarrow 4)(4 \rightarrow 3)(2 \rightarrow 4)(4 \rightarrow 1)(3 \rightarrow 4)(4 \rightarrow 2) \\
& =21555 .
\end{aligned}
$$

- If $\Gamma=\Gamma_{2}$, take

$$
\begin{aligned}
\alpha & :=(3 \rightarrow 4)(4 \rightarrow 5)(1 \rightarrow 3)(3 \rightarrow 4)(2 \rightarrow 3)(3 \rightarrow 1)(4 \rightarrow 3)(3 \rightarrow 2) \\
& =21555 .
\end{aligned}
$$

- If $\Gamma=\Gamma_{3}$, take

$$
\alpha:=(3 \rightarrow 4)(2 \rightarrow 3)(1 \rightarrow 2)(3 \rightarrow 1)=2144
$$

- If $\Gamma=\Gamma_{4}$, take

$$
\alpha=(3 \rightarrow 4)(4 \rightarrow 5)(2 \rightarrow 3)(3 \rightarrow 4)(1 \rightarrow 2)(4 \rightarrow 1)=21555
$$

- Assume $\Gamma=\Theta_{k}$ for $k \geq 5$. Consider the following transformation of $[k]$ :

$$
(u \Rightarrow v):=\left(u \rightarrow u_{1}\right) \ldots\left(u_{d-1} \rightarrow v\right),
$$

where $u, u_{1}, \ldots, u_{d-1}, v$ is the unique path from $u$ to $v$ on the cycle $\Theta_{k}$. Take

$$
\begin{aligned}
\alpha:= & (1 \Rightarrow k-3)(k \Rightarrow k-4)(k-1 \Rightarrow 1)(k-2 \Rightarrow k) \\
& (k-3 \Rightarrow k-1)(k-4 \Rightarrow k-2) .
\end{aligned}
$$

Then, $\alpha=(k-1)(k-1) \ldots(k-1) k 1(k-2)$, where $(k-1)$ appears $k-3$ times, has the cyclic component $(k-2, k)$.

Conversely, assume that $D$ satisfies (ii). If $n \leq 3$, it is clear that $\operatorname{cycl}(\alpha)=0$, for all $\alpha \in\langle D\rangle$, so suppose $n \geq 4$. We first obtain some key properties about the strong components of $\bar{D}$.

Claim 2.9 Any strong component of $\bar{D}$ is an undirected path, an undirected cycle of length 3 or 4, or a claw $K_{3,1}$ (i.e. a bipartite undirected graph on [4] $=[3] \cup\{4\}$ ). Moreover, if a strong component of $D$ is not an undirected path, then it is terminal.

Proof Let $C$ be a strong component of $\bar{D}$. Clearly, $C$ is undirected and, by (ii), it cannot contain a cycle of length at least 5 . If $C$ has a cycle of length 3 or 4 , then the whole of $C$ must be that cycle and $C$ is terminal (otherwise, it would contain $\Gamma_{3}$ or $\Gamma_{4}$, respectively). If $C$ has no cycle of length 3 and 4, then $C$ is a tree. It can only be a path or $K_{3,1}$, for otherwise it would contain $\Gamma_{1}$ or $\Gamma_{2}$; clearly, $K_{3,1}$ may only appear as a terminal component. 
Suppose there is $\alpha \in\langle D\rangle$ that has a cyclic orbit ( $\operatorname{socycl}(\alpha) \neq 0$ ). This cyclic orbit must be contained in a strong component $C$ of $\bar{D}$, and Claim 2.9 implies that $C \cong \Gamma$, where $\Gamma \in\left\{K_{3,1}, \bar{\Theta}_{s}, P_{r}: s \in\{3,4\}, r \in \mathbb{N}\right\}$. If $\Gamma=K_{3,1}$ or $\Gamma=\bar{\Theta}_{s}$, then $C$ is a terminal component, so $\alpha$ acts on $C$ as some transformation $\beta \in\langle\Gamma\rangle$; however, it is easy to check that no transformation in $\langle\Gamma\rangle$ has a cyclic orbit. If $\Gamma=P_{r}$, for some $r$, then $\alpha$ acts on $C$ as a partial transformation $\beta$ of $P_{r}$. Since $\left\langle P_{r}\right\rangle=\mathrm{O}_{r}, \beta$ has no cyclic orbit.

We introduce a new property of a connected digraph $D$ :

( $\star$ ) For every strong component $C$ of $D,|C| \leq 2$ if $C$ is non-terminal, and $|C| \leq 3$ if $C$ is terminal.

Lemma 2.10 Let $D$ be a closed connected digraph on $[n]$ satisfying property $(\star)$. The following are equivalent:

(i) D satisfies property $(\star \star)$.

(ii) $D$ has no subdigraph isomorphic to $\Gamma_{1}, \Gamma_{2}, \Gamma_{3}, \Gamma_{4}$, or $\Theta_{k}$, for some $k \geq 5$.

Proof If (i) holds, it is easy to check that $D$ does not contain any subdigraphs isomorphic to $\Gamma_{1}, \Gamma_{2}, \Gamma_{3}, \Gamma_{4}$, or $\Theta_{k}$ for some $k \geq 5$.

Conversely, suppose that (ii) holds. Let $C$ be a strong component of $D$. If $C$ is non-terminal, Lemma 2.2 implies that $C$ is complete; hence, $|C| \leq 2$ as otherwise $D$ would contain $\Gamma_{4}$ as a subdigraph. If $C$ is terminal, Lemma 2.2 implies that $C$ is complete or $P_{3}$; hence, $|C| \leq 3$ as otherwise $D$ would contain $\Gamma_{3}$ as a subdigraph.

Theorem 2.11 Let $D$ be a connected digraph on $[n]$. The following are equivalent:

(i) For all $\alpha \in\langle D\rangle, \ell(D, \alpha)=n-\operatorname{fix}(\alpha)$.

(ii) $D$ is closed satisfying properties $(\star)$ and $(\star \star)$.

Proof Clearly, $D$ satisfies (i) if and only if it satisfies condition (C1) and $\operatorname{cycl}(\alpha)=0$, for all $\alpha \in\langle D\rangle$. By Theorem 2.5 and Lemmas 2.8 and 2.10, $D$ satisfies (i) if and only if $D$ satisfies (ii).

\subsection{Digraphs satisfying condition (C3)}

The following result characterises digraphs satisfying condition (C3).

Theorem 2.12 Let $D$ be a connected digraph on $[n]$. The following are equivalent:

(i) For every $\alpha \in\langle D\rangle, \ell(D, \alpha)=n-\operatorname{rk}(\alpha)$.

(ii) $\langle D\rangle$ is a band, i.e. every $\alpha \in\langle D\rangle$ is idempotent.

(iii) Either $n=2$ and $D \cong K_{2}$, or there exists a bipartition $V_{1} \cup V_{2}$ of $[n]$ such that $\left(i_{1}, i_{2}\right) \in E(D)$ only if $i_{1} \in V_{1}, i_{2} \in V_{2}$.

Proof Clearly (i) implies (ii): if $\ell(D, \alpha)=n-\operatorname{rk}(\alpha)$, then $\operatorname{rk}(\alpha)=f i x(\alpha)$ by inequality (1), so $\alpha$ is idempotent.

Now we prove that (ii) implies (iii). If there exist $u, v, w \in[n]$ pairwise distinct such that $(u, v),(v, w) \in E(D)$, then $\alpha=(v \rightarrow w)(u \rightarrow v)$ is not an idempotent. 
Therefore, for $n \geq 3$, if every $\alpha \in\langle D\rangle$ is idempotent, then a vertex in $D$ either has in-degree zero or out-degree zero: this corresponds to the bipartition of $[n]$ into $V_{1}$ and $V_{2}$.

We finally prove that (iii) implies (i). Let $n \geq 3$ and suppose that there exists a bipartition $V_{1} \cup V_{2}$ of $[n]$ such that $\left(i_{1}, i_{2}\right) \in E(D)$ only if $i_{1} \in V_{1}, i_{2} \in V_{2}$. Then for any $\alpha \in\langle D\rangle$, all elements of $V_{2}$ are fixed by $\alpha$ and $i_{1} \alpha \in\left\{i_{1}\right\} \cup N^{+}\left(i_{1}\right)$ for any $i_{1} \in V_{1}$. In particular, any non-fixed point of $\alpha$ is mapped to a fixed point, so $r:=\operatorname{rk}(\alpha)=\operatorname{fix}(\alpha)$. Let $J:=\left\{v_{1}, \ldots, v_{n-r}\right\} \subseteq V_{1}$ be the set of non-fixed points of $\alpha$; therefore

$$
\alpha=\left(v_{1} \rightarrow v_{1} \alpha\right) \ldots\left(v_{n-r} \rightarrow v_{n-r} \alpha\right),
$$

where each one of the $n-r$ arcs above belongs to $\langle D\rangle$. The result follows by inequality (1).

\section{Arc-generated semigroups with long words}

Fix $n \geq 2$. In this section, we consider digraphs $D$ that maximise $\ell(D, r)$ and $\ell(D)$. For $r \in[n-1]$, define

$$
\begin{aligned}
\ell_{\max }(n, r) & :=\max \{\ell(D, r): V(D)=[n]\}, \\
\ell_{\max }(n) & :=\max \{\ell(D): V(D)=[n]\} .
\end{aligned}
$$

The first few values of $\ell_{\max }(n, r)$, calculated with the GAP package Semigroups [7], are given in Table 1. By Lemma 2.1, $\ell_{\max }(n, 1)=n-1$ for all $n \geq 2$; henceforth, we shall always assume that $n \geq 3$ and $r \in[n-1] \backslash\{1\}$.

In the following sections, we restrict the class of digraphs that we consider in the definition of $\ell_{\max }(n, r)$ and $\ell_{\max }(n)$ to two important cases: acyclic digraphs and strong tournaments.

\subsection{Acyclic digraphs}

For any $n \geq 3$, let Acyclic $_{n}$ be the set of all acyclic digraphs on $[n]$, and, for any $r \in[n-1]$, define

Table 1 First values of $\ell_{\max }(n, r)$

\begin{tabular}{rrrrrr}
\hline$n$ & $r$ & & & & \\
\cline { 2 - 5 }$n$ & 1 & 2 & 3 & 4 & 5 \\
\hline 2 & 1 & & & & \\
3 & 2 & 6 & & & \\
4 & 3 & 11 & 13 & & \\
5 & 4 & 18 & 24 & 33 & \\
6 & 5 & 26 & 42 & 51 & 66 \\
\hline
\end{tabular}




$$
\begin{aligned}
\ell_{\max }^{\operatorname{Acyclic}}(n, r) & :=\max \left\{\ell(A, r): A \in \operatorname{Acyclic}_{n}\right\}, \\
\ell_{\max }^{\operatorname{Acyclic}}(n): & =\max \left\{\ell(A): A \in \operatorname{Acyclic}_{n}\right\} .
\end{aligned}
$$

Without loss of generality, we assume that any acyclic digraph $A$ on $[n]$ is topologically sorted, i.e. $(u, v) \in E(A)$ only if $v>u$.

In this section, we establish the following theorem.

Theorem 3.1 For any $n \geq 3$ and $r \in[n-1] \backslash\{1\}$,

$$
\begin{aligned}
\ell_{\max }^{\mathrm{Acyclic}}(n, r) & =\frac{(n-r)(n+r-3)}{2}+1, \\
\ell_{\max }^{\mathrm{Acyclic}}(n) & =\ell_{\max }^{\mathrm{Acyclic}}(n, 2)=\frac{1}{2}\left(n^{2}-3 n+4\right) .
\end{aligned}
$$

First of all, we settle the case $r=n-1$, for which we have a finer result.

Lemma 3.2 Let $n \geq 3$ and $A \in$ Acyclic $_{n}$. Then, $\ell(A, n-1)$ is equal to the length of a longest path in $A$. Therefore,

$$
\ell_{\max }^{\text {Acyclic }}(n, n-1)=n-1 .
$$

Proof Let $v_{1}, \ldots, v_{l+1}$ be a longest path in $A$. Then $\alpha \in\langle A\rangle$ defined by

$$
v \alpha:= \begin{cases}v_{i+1} & \text { if } v=v_{i}, i \in[l] \\ v & \text { otherwise, }\end{cases}
$$

has rank $n-1$ and requires at least $l$ arcs, since it moves $l$ vertices.

Conversely, let $\alpha \in A$ be a transformation of rank $n-1$, and consider a word expressing $\alpha$ in $A^{*}$ :

$$
\alpha=\left(u_{1} \rightarrow v_{1}\right)\left(u_{2} \rightarrow v_{2}\right) \ldots\left(u_{s} \rightarrow v_{s}\right) .
$$

Since $\alpha$ has rank $n-1$, we must have $v_{2}=u_{1}$ and by induction $v_{i}=u_{i-1}$ for $2 \leq i \leq s$. As $A$ is acyclic, $u_{s}, u_{s-1}, \ldots, u_{1}, v_{1}$ forms a path in $A$, so $s \leq l$.

The following lemma shows that the formula of Theorem 3.1 is an upper bound for $\ell_{\max }^{\text {Acyclic }}(n, r)$.

Lemma 3.3 For any $n \geq 3$ and $r \in[n-1] \backslash\{1\}$,

$$
\ell_{\max }^{\text {Acyclic }}(n, r) \leq \frac{(n-r)(n+r-3)}{2}+1
$$

Proof Let $A$ be an acyclic digraph on [n], let $\alpha \in\langle A\rangle$ be a transformation of rank $r \geq 2$, and let $L \subset V(A)$ be the set of terminal vertices of $A$. For any $u, v \in[n]$, denote the length of a longest path from $u$ to $v$ in $A$ as $\psi_{A}(u, v)$. 
Claim $3.4 \ell(A, \alpha) \leq \sum_{v \in[n]} \psi_{A}(v, v \alpha)$.

Proof Let $\omega=\left(a_{1} \rightarrow b_{1}\right) \ldots\left(a_{l} \rightarrow b_{l}\right)$ be a shortest word expressing $\alpha$ in $A^{*}$, with $l=\ell(A, \alpha)$. Say that the arc $\left(a_{i} \rightarrow b_{i}\right), i \geq 2$, carries $v \in[n]$ if $v\left(a_{1} \rightarrow\right.$ $\left.b_{1}\right) \ldots\left(a_{i-1} \rightarrow b_{i-1}\right)=a_{i}$ (assume that $a_{1} \rightarrow b_{1}$ only carries $\left.a_{1}\right)$. Every arc $\left(a_{i} \rightarrow\right.$ $b_{i}$ ) carries at least one vertex, for otherwise we could remove that arc form the word $\omega$ and obtain a shorter word still expressing $\alpha$. Let $v \in[n]$, and denote $v_{0}=v$ and $v_{i}=v\left(a_{1} \rightarrow b_{1}\right) \ldots\left(a_{i} \rightarrow b_{i}\right)$ (and hence $v_{l}=v \alpha$ ). Let us remove the repetitions in this sequence: let $j_{0}=0$ and for $i \geq 1, j_{i}=\min \left\{j: v_{j} \neq v_{j_{i-1}}\right\}$. Then the sequence $v=v_{j_{0}}, v_{j_{1}}, \ldots, v_{j_{l(v)}}=v \alpha$ forms a path in $A$ of length $l(v)$, and hence $l(v) \leq \psi(v, v \alpha)$. For each $v \in[n]$, there are $l(v)$ arcs in $\omega$ carrying $v$, so the length of $\omega$ satisfies

$$
l \leq \sum_{v=1}^{n} l(v) \leq \sum_{v \in[n]} \psi_{A}(v, v \alpha)
$$

Claim 3.5 If $|L| \geq 2$, then $\sum_{v \in[n]} \psi_{A}(v, v \alpha) \leq \frac{(n-r)(n+r-3)}{2}$.

Proof As $|L| \geq 2$, and $A$ is topologically sorted, we have $\{n, n-1\} \subseteq L$, and any $\alpha \in\langle A\rangle$ fixes both $n-1$ and $n$, i.e. $\psi_{A}(v, v \alpha)=0$ for $v \in\{n-1, n\}$. For any $v \in[n-2]$, we have

$$
\psi_{A}(v, v \alpha) \leq \min \{n-1, v \alpha\}-v
$$

Hence

$$
\begin{aligned}
\sum_{v \in[n]} \psi_{A}(v, v \alpha) & =\sum_{v \in[n-2]} \psi_{A}(v, v \alpha) \\
& \leq \sum_{v \in[n-2]}(\min \{n-1, v \alpha\}-v) \\
& =\sum_{w \in[n-2] \alpha}\left(\min \{n-1, w\}\left|w \alpha^{-1}\right|\right)-T_{n-2}
\end{aligned}
$$

where $T_{k}=\frac{k(k+1)}{2}$. The summation is maximised when $\left|n \alpha^{-1}\right|=n-r$ and $\left|w \alpha^{-1}\right|=$ 1 for $n-r+1 \leq w \leq n-2$, thus yielding

$$
\begin{aligned}
\sum_{v \in[n]} \psi_{A}(v, v \alpha) & \leq(n-1)(n-r)+\left(T_{n-2}-T_{n-r}\right)-T_{n-2} \\
& =\frac{(n-r)(n+r-3)}{2} .
\end{aligned}
$$

Claim 3.6 If $|L|=1$, then $\ell(A, \alpha) \leq \frac{(n-r)(n+r-3)}{2}+1$. 
Proof As $A$ is topologically sorted, $L=\{n\}$. We use the notation from the proof of Claim 3.4. We then have $l(n)=0$. We have three cases:

Case $1(n-1)$ is fixed by $\alpha$. Then, $l(n-1)=0$ and $l(v) \leq \min \{n-1, v \alpha\}-v$ for all $v \in[n-2]$. By the same reasoning as in Claim 3.5, we obtain $\ell(A, \alpha) \leq$ $\frac{(n-r)(n+r-3)}{2}$.

Case $2(n-1) \alpha=n$ and $v \alpha \leq n-1$ for every $v \in[n-2]$. Then again $l(v) \leq$ $\min \{n-1, v \alpha\}-v$, for all $v \in[n-2]$, and $\ell(A, \alpha) \leq \frac{(n-r)(n+r-3)}{2}$.

Case $3 n$ has at least two pre-images under $\alpha$. Let $\omega=\left(a_{1} \rightarrow b_{1}\right) \ldots\left(a_{l} \rightarrow b_{l}\right)$ be a shortest word expressing $\alpha$ in $A^{*}$, and denote $\alpha_{0}=$ id and $\epsilon_{i}=\left(a_{i} \rightarrow b_{i}\right)$, $\alpha_{i}=\epsilon_{1} \ldots \epsilon_{i}$ for $i \in[l]$. We partition $n \alpha^{-1}$ into two parts $S$ and $T$ :

$$
S=\left\{v \in n \alpha^{-1}: v_{l(v)-1}=n-1\right\}, \quad T=n \alpha^{-1} \backslash S .
$$

For all $v \in S$, if the arc carrying $v$ to $n-1$ is $\epsilon_{j}$, then $(n-1) \alpha_{j-1}^{-1} \subseteq S$ ( $v$ can only collapse with other pre-images of $\alpha$ ). Then the $\operatorname{arc}(n-1 \rightarrow n)$ occurs only once in the word $\omega$ (if it occurs multiple times, then remove all but the last occurrence of that arc to obtain a shorter word expressing $\alpha$ ). If we do not count that arc, we have $l^{\prime}(v) \leq n-1-v$ arcs carrying $v$ if $v \in S$, $l(v) \leq n-1-v$ arcs carrying $v$ if $v \in T$, and $l(v) \leq v \alpha-v$ if $v \alpha \neq n$. Again, we obtain $\ell(A, \alpha) \leq \frac{(n-r)(n+r-3)}{2}+1$.

Lemma 3.3 follows by the previous claims.

The following lemma completes the proof of Theorem 3.1.

Lemma 3.7 For any $n \geq 3$ and $r \in[n-1] \backslash\{1\}$, there exists an acyclic digraph $Q_{n}$ on $[n]$ and a transformation $\beta_{r} \in\left\langle Q_{n}\right\rangle$ of rank $r$ such that

$$
\ell\left(Q_{n}, \beta_{r}\right) \geq \frac{(n-r)(n+r-3)}{2}+1 .
$$

Proof Let $Q_{n}$ be the acyclic digraph on $[n]$ with edge set

$$
E\left(Q_{n}\right):=\{(u, u+1): u \in[n-1]\} \cup\{(n-2, n)\} .
$$

For any $r \in[n-1] \backslash\{1\}$, define $\beta_{r} \in\left\langle Q_{n}\right\rangle$ by

$$
v \beta_{r}:=\left\{\begin{array}{lll}
n-r+v & \text { if } v \in[r-2], \\
n-1 & \text { if } v \in[n-1] \backslash[r-2], n-v \equiv 0 \quad \bmod 2, \\
n & \text { if } v \in[n-1] \backslash[r-2], n-v \equiv 1 \quad \bmod 2, \\
n & \text { if } v=n . &
\end{array}\right.
$$

Let $\beta_{r}$ be expressed as a word in $Q_{n}^{*}$ of minimum length as

$$
\beta_{r}=\left(a_{1} \rightarrow b_{1}\right) \ldots\left(a_{l} \rightarrow b_{l}\right),
$$

where $l=\ell\left(Q_{n}, \beta_{r}\right)$. Denote $\alpha_{0}:=\mathrm{id}, \epsilon_{i}:=\left(a_{i} \rightarrow b_{i}\right)$, and $\alpha_{i}:=\epsilon_{1} \ldots \epsilon_{i}$, for $i \in[l]$. Say that $\epsilon_{i}$ carries $u \in[n]$ if $u \alpha_{i-1}=a_{i}$ and hence $u \alpha_{i} \neq u \alpha_{i-1}$. 
Claim 3.8 For each $i \in[l]$, the arc $\epsilon_{i}$ carries exactly one vertex.

Proof First, $\left(a_{1}, b_{1}\right) \in E\left(Q_{n}\right)$ and $a_{1} \beta_{r}=b_{1} \beta_{r}$ imply that $a_{1}=n-1$ and $b_{1}=n$. Suppose that there is an arc $\epsilon_{j}, j \in[l]$, that carries two vertices $u<v$; take $j$ to be minimal index with this property. We remark that $v \leq n-2$ and $u \alpha_{j-1}=v \alpha_{j-1}$ imply $u \beta_{r}=v \beta_{r}$. Then $w:=u+1$ satisfies $w \beta_{r} \neq u \beta_{r}$, so $w$ is not carried by $\epsilon_{j}$. If $w \alpha_{j-1} \leq n-2$, then $u \alpha_{j-1}<w \alpha_{j-1}<v \alpha_{j-1}$ since $u<w<v$ and the graph induced by $[n-2]$ in $Q_{n}$ is the directed path $\vec{P}_{n-2}$; this contradicts that $u \alpha_{j-1}=v \alpha_{j-1}$. Hence $w \alpha_{j-1} \geq n-1$ and $v \alpha_{j-1} \geq n-1$. If $v \alpha_{j-1}=n$ or $v \beta_{r}=n-1$, then $\epsilon_{j}$ does not carry $v$. Thus, $v \alpha_{j-1}=n-1$ and $v \beta_{r}=n$. Then, in order to carry $v$ to $n-1$, we have $\epsilon_{s}=(n-2 \rightarrow n-1)$ for at least one $s \in[l]$, and $\epsilon_{j}=(n-1 \rightarrow n)$. For $s \in[j-1]$, replace all occurrences $\epsilon_{s}=(n-2 \rightarrow n-1)$ with $\epsilon_{s}^{\prime}:=(n-2 \rightarrow n)$ and delete $\epsilon_{j}$ : this yields a word in $Q_{n}^{*}$ of length $l^{\prime}<l$ expressing $\beta_{r}$, which is a contradiction.

For all $i \in[l]$, denote $\delta(i):=\sum_{v \in[n]} d_{Q_{n}}\left(v \alpha_{i}, v \beta_{r}\right)$. We then have $\delta(l)=0$, and by the claim, $\delta(i) \geq \delta(i-1)-1$ for all $i \in[l]$. Thus $l \geq \delta(0)$, where

$$
\begin{aligned}
\delta(0) & =\sum_{v \in[n]} d_{Q_{n}}\left(v, v \beta_{r}\right) \\
& =\sum_{v=1}^{r-2}(n-r)+\sum_{v=r-1}^{n-2}(n-1-v)+1 \\
& =\frac{(n-r)(n+r-3)}{2}+1 .
\end{aligned}
$$

\subsection{Strong tournaments}

Let $n \geq 3$. Recall that if $T$ is a strong tournament on $[n]$, then $\{a \rightarrow b:(a, b) \in E(T)\}$ is a minimal generating set of $\operatorname{Sing}_{n}$. Let Tour $_{n}$ denote the set of all strong tournaments on $[n]$. For $r \in[n-1]$, define

$$
\begin{gathered}
\ell_{\max }^{\operatorname{Tour}}(n, r):=\max \left\{\ell(T, r): T \in \operatorname{Tour}_{n}\right\}, \\
\ell_{\max }^{\operatorname{Tour}}(n):=\max \left\{\ell(T): T \in \operatorname{Tour}_{n}\right\} .
\end{gathered}
$$

Define analogously $\ell_{\min }^{\text {Tour }}(n, r)$ and $\ell_{\min }^{\text {Tour }}(n)$. The first few values of $\ell_{\min }^{\text {Tour }}(n, r)$ and $\ell_{\max }^{\text {Tour }}(n, r)$, calculated with the GAP package Semigroups [7] using data from [6], are given in Table 2. The calculation of these values has been the inspiration for the results of this section and the conjectures of the next one.

Lemma 3.9 Let $n \geq 3$ and $T \in \operatorname{Tour}_{n}$.

1. For any partition $P$ of $[n]$ into $r$ parts, there exists an idempotent $\alpha \in \operatorname{Sing}_{n}$ with $\operatorname{ker}(\alpha)=P$ such that $\ell(T, \alpha)=n-r$. 
Table 2 First values of $\left(\ell_{\min }^{\text {Tour }}(n, r), \ell_{\max }^{\text {Tour }}(n, r)\right)$

\begin{tabular}{llllll}
\hline & $r$ & & & & \\
$n$ & 2 & 3 & 4 & 5 & 6 \\
\hline 3 & $(6,6)$ & & & & \\
4 & $(8,8)$ & $(11,11)$ & & & \\
5 & $(6,11)$ & $(8,14)$ & $(10,17)$ & & \\
6 & $(8,13)$ & $(10,18)$ & $(11,21)$ & $(13,24)$ & \\
7 & $(8,16)$ & $(10,22)$ & $(11,26)$ & $(13,29)$ & $(15,32)$ \\
\hline
\end{tabular}

2. For any $r$-subset $S$ of $[n]$, there exists an idempotent $\alpha \in \operatorname{Sing}_{n}$ with $\operatorname{Im}(\alpha)=S$ such that $\ell(T, \alpha)=n-r$.

Proof 1 . Let $P=\left\{P_{1}, \ldots, P_{r}\right\}$. For all $1 \leq i \leq r$, the digraph $T\left[P_{i}\right]$ induced by $P_{i}$ is a tournament, so it is connected and there exists a vertex $v_{i}$ reachable by any other vertex in $P_{i}$ : let $\alpha$ map the whole of $P_{i}$ to $v_{i}$. Then $\alpha$, when restricted to $P_{i}$, is a constant map, which can be computed using $\left|P_{i}\right|-1$ arcs. Summing for $i$ from 1 to $r$, we obtain that $\ell(T, \alpha)=n-r$.

2. Without loss of generality, let $S=[r] \subseteq[n]$. For every $v \in[n]$, define

$$
s(v):=\min \left\{s \in S: d_{T}\left(s^{\prime}, v\right) \geq d_{T}(s, v), \forall s^{\prime} \in S\right\} .
$$

In particular, if $v \in S$, then $s(v)=v$. Moreover, if $v=v_{0}, v_{1}, \ldots, v_{d}=s(v)$ is a shortest path from $v$ to $s(v)$, with $d=d_{T}(v, s(v))$, then $s\left(v_{i}\right)=s(v)$ for all $0 \leq i \leq d$. For each $v \in[n]$, fix a shortest path $P_{v}$ from $v$ to $s(v)$, and consider the digraph $D$ on $[n]$ with edges

$$
E(D):=\left\{(a, b):(a, b) \in E\left(P_{v}\right) \text { for some } v \in[n]\right\} \text {. }
$$

Then, $D$ is acyclic and the set of vertices with out-degree zero in $D$ is exactly $S$. Let sort $[n]$ so that $D$ has reverse topological order: $(a, b) \in E(D)$ only if $a>b$. Note that $S$ is fixed by this sorting. Let $\alpha$ be given by $v \alpha:=s(v)$; hence, with the above sorting

$$
\alpha=\bigcirc_{v=n}^{r+1}\left(v \rightarrow v_{1}\right)
$$

Lemma 3.10 Let $n \geq 3, T \in \operatorname{Tour}_{n}$, and $\alpha:=(u \rightarrow v) \in \operatorname{Sing}_{n}$, for $(u, v) \notin E(T)$. Then

$$
\ell(T, \alpha)=4 d_{T}(u, v)-2 .
$$

Proof Let $u=v_{0}, v_{1}, \ldots, v_{d}=v$ be a shortest path from $u$ to $v$ in $T$, where $d:=$ $d_{T}(u, v)$. As $(u, v) \notin E(T)$ and $T$ is a tournament, we must have $(v, u) \in E(T)$. 
Fig. 2 Circulant tournament $\kappa_{5}$

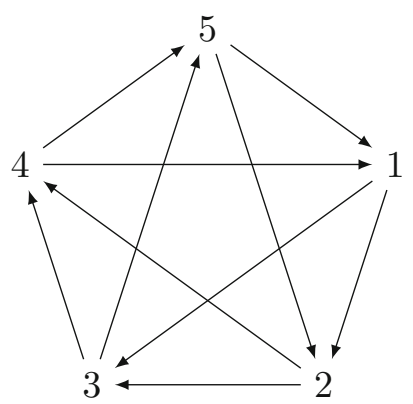

By the minimality of the path, for any $j+1<i$, we have $\left(v_{j}, v_{i}\right) \notin E(T)$, so $\left(v_{i}, v_{j}\right) \in E(T)$. Then, the following expresses $\alpha$ with arcs in $T^{*}$ :

$$
\begin{aligned}
\left(v_{0} \rightarrow v_{d}\right)= & \left(v_{d} \rightarrow v_{0}\right)\left(v_{d-1} \rightarrow v_{d}\right)\left(v_{d-2} \rightarrow v_{d-1}\right) \cdots\left(v_{1} \rightarrow v_{2}\right)\left(v_{0} \rightarrow v_{1}\right) \\
& \left(\left(v_{2} \rightarrow v_{0}\right)\left(v_{1} \rightarrow v_{2}\right)\right)\left(\left(v_{3} \rightarrow v_{1}\right)\left(v_{2} \rightarrow v_{3}\right)\right) \\
& \cdots\left(\left(v_{d} \rightarrow v_{d-2}\right)\left(v_{d-1} \rightarrow v_{d}\right)\right) \\
& \left(v_{d-2} \rightarrow v_{d-1}\right) \cdots\left(v_{0} \rightarrow v_{1}\right) .
\end{aligned}
$$

So $\ell(T, \alpha) \leq 4 d-2$. For the lower bound, we note that any word in $T^{*}$ expressing $(u \rightarrow v)$ must begin with $(v \rightarrow u)$. Then, $u$ has to follow a walk in $T$ towards $v$; say this walk has length $l \geq d$. All the vertices on the walk must be moved away (as otherwise they would collapse with $u$ ) and have to come back to their original position (since $\alpha$ fixes them all); as the shortest cycle in a tournament has length 3 , this process adds at least $3(l-1)$ symbols to the word. Altogether, this yields a word of length at least

$$
1+l+3(l-1)=4 l-2 \geq 4 d-2 .
$$

Let $n=2 m+1 \geq 3$ be odd, and let $\kappa_{n}$ be the circulant tournament on $[n]$ with edges $E\left(\kappa_{n}\right):=\{(i,(i+j) \bmod n): i \in[n], j \in[m]\}$. Figure 2 illustrates $\kappa_{5}$. In the following theorem, we use $\kappa_{n}$ to provide upper and lower bounds for $\ell_{\min }^{\text {Tour }}(n, r)$ and $\ell_{\max }^{\text {Tour }}(n, r)$ when $n$ is odd.

Theorem 3.11 For any $n$ odd, we have

$$
\begin{aligned}
n+r-2 & \leq \ell_{\min }^{\operatorname{Tour}}(n, r) \leq n+8 r, \\
(\hat{r}+1)(n-\hat{r})-1 & \leq \ell_{\max }^{\operatorname{Tour}}(n, r) \leq 6 r n+n-10 r .
\end{aligned}
$$

where $\hat{r}=\min \{r-1,\lfloor n / 2\rfloor\}$. 
Proof Let $T \in \operatorname{Tour}_{n}$ and $2 \leq r \leq n-1$. We introduce the following notation:

$$
\begin{aligned}
{[n]_{r} } & :=\left\{\mathbf{u}:=\left(u_{1}, \ldots, u_{r}\right): u_{i} \neq u_{j}, \forall i, j\right\}, \\
\Delta(T, r) & :=\max \left\{\sum_{i=1}^{r} d_{T}\left(u_{i}, v_{i}\right): \mathbf{u}, \mathbf{v} \in[n]_{r}\right\} .
\end{aligned}
$$

The result follows by the next claims.

Claim 3.12 $r^{\prime}\left(\operatorname{diam}(T)-r^{\prime}+1\right)+r-r^{\prime} \leq \Delta(T, r) \leq r \operatorname{diam}(T)$, where $r^{\prime}=$ $\min \{r,\lfloor(\operatorname{diam}(T)+1) / 2\rfloor\}$.

Proof The upper bound is clear. For the lower bound, let $u, v \in[n]$ be such that $d_{T}(u, v)=\operatorname{diam}(T)$, and let $u=v_{0}, v_{1}, \ldots, v_{d}=v$ be a shortest path from $u$ to $v$, where $d=\operatorname{diam}(T)$. Then, $d_{T}\left(v_{i}, v_{j}\right)=j-i$, for all $0 \leq i \leq j \leq D$. If $1 \leq r \leq\lfloor(d+1) / 2\rfloor$, consider $\mathbf{u}^{\prime}=\left(v_{0}, \ldots, v_{r-1}\right)$ and $\mathbf{v}^{\prime}=\left(v_{d-r+1}, \ldots, v_{d}\right)$, so we obtain $\Delta(T, r) \geq r(d-r+1)$. If $r \geq\lfloor(d+1) / 2\rfloor$, simply add vertices $u_{j}^{\prime}$ and $v_{j}^{\prime}$ such that $\left(u_{j}^{\prime}, v_{j}^{\prime}\right) \notin T$.

Claim 3.13 $\min \{\Delta(T, r): T \in \operatorname{Tour}(n)\}=\Delta\left(\kappa_{n}, r\right)=2 r$.

Proof Let $\mathbf{u}=\left(u_{1}, \ldots, u_{n}\right)$ form a Hamiltonian cycle, and choose $\mathbf{v}=\left(u_{n}, u_{1}, \ldots\right.$, $\left.u_{n-1}\right)$. Then $d_{T}\left(u_{i}, v_{i}\right) \geq 2$ for all $i$. Conversely, since $\operatorname{diam}\left(\kappa_{n}\right)=2$, we have $\Delta\left(\kappa_{n}, r\right)=2 r$.

Claim 3.14 $n-r+\Delta(T, r-1) \leq \ell(T, r) \leq n+6 r \operatorname{diam}(T)-4 r$.

Proof For the lower bound, consider $\alpha \in \operatorname{Sing}_{n}$ as follows. Let $\mathbf{u}=\left(u_{1}, \ldots, u_{r-1}\right)$ and $\mathbf{v}=\left(v_{1}, \ldots, v_{r-1}\right)$ achieve $\Delta(T, r-1)$, and let $v \notin\left\{v_{1}, \ldots, v_{r-1}\right\}$; define

$$
x \alpha= \begin{cases}v_{i} & \text { if } x=u_{i} \\ v & \text { otherwise }\end{cases}
$$

Let $\omega=e_{1} \ldots e_{l}$ (where $e_{i}=\left(a_{i} \rightarrow b_{i}\right)$ ) be a shortest word expressing $\alpha$, where $l:=\ell(T, \alpha)$. Recall that an arc $e_{i}$ carries a vertex $c$ if $c e_{1} \ldots e_{i-1}=a_{i}$. By the minimality of $\omega$, every arc carries at least one vertex. Moreover, if $c$ and $d$ are carried by $e_{i}$, then $c \alpha=d \alpha$; therefore, we can label every $\operatorname{arc} e_{i}$ of $\omega$ by an element $c\left(e_{i}\right) \in \operatorname{Im}(\alpha)$ if $e_{i}$ carries vertices eventually mapping to $c\left(e_{i}\right)$. Denote the number of arcs labelled $c$ as $l(c)$, we then have $l=\sum_{c \in \operatorname{Im}(\alpha)} l(c)$. For any $u \in V$, there are at least $d_{T}(u, u \alpha)$ arcs carrying $u$. Therefore,

$$
l=\sum_{c \in \operatorname{Im}(\alpha)} l(c) \geq \sum_{i=1}^{r-1} d_{T}\left(u_{i}, v_{i}\right)+\sum_{a \notin \mathbf{u}} d_{T}(a, v) \geq \Delta(T, r-1)+n-r
$$

For the upper bound, we can express any $\alpha \in \operatorname{Sing}_{n}$ of rank $r$ in the following fashion. By Lemma 3.9, there exists $\beta \in \operatorname{Sing}_{n}$ with the same kernel as $\alpha$ such that 
$\ell(T, \beta)=n-r$. Suppose that $\operatorname{Im}(\alpha)=\left\{v_{1}, \ldots, v_{r}\right\}$ and $\operatorname{Im}(\beta)=\left\{u_{1}, \ldots, u_{r}\right\}$, where $u_{i} \beta^{-1}=v_{i} \alpha^{-1}$, for $i \in[r]$. Let $h \in[n] \backslash \operatorname{Im}(\beta)$. Define a transformation $\gamma$ of $[n]$ by

$$
x \gamma= \begin{cases}v_{i} & \text { if } x=u_{i} \\ v_{1} & \text { if } x=h \\ x & \text { otherwise }\end{cases}
$$

Then $\alpha=\beta \gamma$, where $\gamma \in \operatorname{Sing}_{n}$, and by Theorem 1.1

$$
\ell\left(K_{n}, \gamma\right)=n-\operatorname{fix}(\gamma)+\operatorname{cycl}(\gamma) \leq r+\frac{r}{2}=\frac{3 r}{2} .
$$

By Lemma 3.10, each arc associated with $K_{n}$ may be expressed in at most 4diam $(T)-2$ arcs associated with $T$; therefore,

$$
\ell(T, \gamma) \leq \frac{3 r}{2}(4 \operatorname{diam}(T)-2)=6 r \operatorname{diam}(T)-3 r
$$

Thus,

$$
\ell(T, \alpha) \leq \ell(T, \beta)+\ell(T, \gamma) \leq n+6 r \operatorname{diam}(T)-4 r .
$$

\section{Conjectures and open problems}

We finish the paper by proposing few conjectures and open problems.

Let $\pi_{n}$ be the tournament on $[n]$ with edges $E\left(\pi_{n}\right):=\{(i,(i+1) \bmod n): i \in$ $[n]\} \cup\{(i, j): j+1<i\}$. Figure 3 illustrates $\pi_{5}$.

Conjecture 4.1 For every $n \geq 3, r \in[n-1]$, and $T \in \operatorname{Tour}_{n}$, we have

$$
\ell(T, r) \leq \ell\left(\pi_{n}, r\right)=\ell_{\max }^{\text {Tour }}(n, r),
$$

with equality if and only if $T \cong \pi_{n}$. Furthermore,

$$
\ell\left(\pi_{n}\right)=\ell_{\max }^{\text {Tour }}(n)=\frac{n^{2}+3 n-6}{2},
$$

Fig. $3 \pi_{5}$

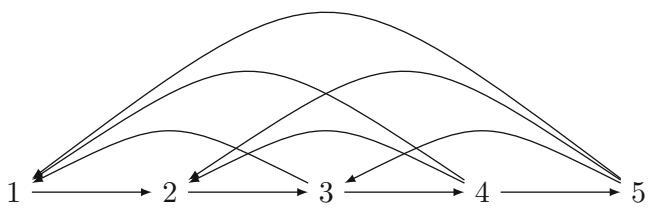


which is achieved for $\alpha:=n(n-1) \ldots 2 n$.

Tournament $\pi_{n}$ has appeared in the literature before: it is shown in [8] that $\pi_{n}$ has the minimum number of strong subtournaments among all strong tournaments on $[n]$. On the other hand, it was shown in [1] that, for $n$ odd, the circulant tournament $\kappa_{n}$ has the maximal number of strong subtournaments among all strong tournaments on $[n]$.

Conjecture 4.2 For every $n \geq 3$ odd, $r \in[n-1]$, and $T \in \operatorname{Tour}_{n}$, we have

$$
\ell_{\min }^{\text {Tour }}(n, r)=\ell\left(\kappa_{n}, r\right)
$$

Furthermore,

$$
\ell_{\min }^{\text {Tour }}(n, 2)=n+1 \text { and } \ell_{\min }^{\text {Tour }}(n, r)=n+r \text {, }
$$

for all $3 \leq r \leq \frac{n+1}{2}$.

Conjecture 4.3 There exists $c>0$ such that for every simple digraph $D$ on $[n]$, $\ell(D)=O\left(n^{c}\right)$.

The referee of this paper noted that the automorphism groups of $K_{n}$ and $\left\langle K_{n}\right\rangle=$ $\operatorname{Sing}_{n}$ are both isomorphic to $\mathrm{Sym}_{n}$ and proposed the following problems.

Problem 1 Investigate connections between the automorphism groups of $D$ and $\langle D\rangle$. Is it possible to classify all digraphs $D$ such that the automorphism group of $D$ and of $\langle D\rangle$ are isomorphic?

Problem 2 Generalise the ideas of this paper to oriented matroids. Is there a natural way to associate (not necessarily idempotent) transformations to each signed circuit of an oriented matroid?

In a forthcoming paper, we investigate the relationship between the graph theoretic properties of $D$ and the semigroup properties of $\langle D\rangle$.

Acknowledgements The second and third authors were supported by the EPSRC grant EP/K033956/1. We kindly thank the insightful comments and suggestions for open problems of the anonymous referee of this paper.

Open Access This article is distributed under the terms of the Creative Commons Attribution 4.0 International License (http://creativecommons.org/licenses/by/4.0/), which permits unrestricted use, distribution, and reproduction in any medium, provided you give appropriate credit to the original author(s) and the source, provide a link to the Creative Commons license, and indicate if changes were made.

\section{References}

1. Beineke, L.W., Harary, F.: The maximum number of strongly connected subtournaments. Can. Math. Bull. 8, 491-498 (1965)

2. Howie, J.M.: The subsemigroup generated by the idempotents of a full transformation semigroup. J. Lond. Math. Soc. 41, 707-716 (1966) 
3. Howie, J.M.: Idempotent generators in finite full transformation semigroups. Proc. R. Soc. Edinb. 81A, 317-323 (1978)

4. Howie, J.M.: Products of idempotents in finite full transformation semigroups. Proc. R. Soc. Edinb. 86A, 243-254 (1980)

5. Iwahori, N.: A length formula in a semigroup of mappings. J. Fac. Sci. Univ. Tokyo Sect. 1A Math. 24, 255-260 (1977)

6. McKay, B.: Catalogue of Directed Graphs. September 2015. Retrieved from https://cs.anu.edu.au/ people/Brendan.McKay/data/digraphs.html

7. Mitchell, J.D., et al.: Semigroups-GAP Package, Version 3.0. September 2015

8. Moon, J.W.: On subtournaments of a tournament. Can. Math. Bull. 9, 297-301 (1966)

9. Solomon, A.: Catalan monoids, monoids of local endomorphisms, and their presentations. Semigroup Forum 53, 351-368 (1996)

10. You, T., Yang, X.: A classification of the maximal idempotent-generated subsemigroups of finite singular groups. Semigroup Forum 64, 236-242 (2002)

11. Yang, X., Yang, H.: Maximal regular subsemibands of $\operatorname{Sing}_{n}$. Semigroup Forum 72, 75-93 (2006)

12. Yang, X., Yang, H.: Isomorphisms of transformation semigroups associated with simple digraphs. Asian Eur. J. Math. 2(4), 727-737 (2009) 